\title{
EVALUACIÓN DE VARIABLES PSICOLÓGICAS EN EL TRATAMIENTO DE PERSONAS CON ADICCIÓN: PROPIEDADES PSICOMÉTRICAS DEL CUESTIONARIO VIP
}

\author{
ASSESSMENT OF PSYCHOLOGICAL VARIABLES IN TREATING \\ PEOPLE WITH ADDICTION: PSYCHOMETRIC PROPERTIES OF \\ THE QUESTIONNAIRE VIP
}

\author{
EdUARDO J. PEDRERo-PÉREZ
}

Cómo referenciar este artículo/How to reference this article:

Pedrero-Pérez, E. J. (2016). Evaluación de variables psicológicas en el tratamiento de personas con adicción: propiedades psicométricas del cuestionario VIP [Assessment of Psychological Variables in Treating People with Addiction: Psychometric Properties of the Questionnaire VIP]. Acción Psicológica, 13(1), 91-106. http://dx.doi.org/10.5944/ap.13.1.15363

\section{Resumen}

El Cuestionario de Variables de Interacción Psicosocial fue propuesto hace una década para explorar variables psicológicas críticamente relacionadas con la adicción: autoestima, autoeficacia general, optimismo, locus de control, habilidades sociales, autocontrol y estilos de afrontamiento. En el presente estudio se ha realizado un análisis factorial confirmatorio sobre una muestra de 1242 sujetos en tratamiento por adicción, y se ha estudiado la fiabilidad de las escalas y su validez predictiva sobre variables de salud y estrés percibido. El estudio factorial mostró una adecuada validez de constructo y todas las escalas han mostrado adecuada fiabilidad, salvo la de locus de control, con sólo tres ítems. Varias escalas muestran correlaciones de gran magnitud con estrés percibido, calidad de vida relacionada con la salud y riesgo de mala salud mental. Destaca la capacidad predictiva de la escala de optimismo sobre estas tres variables, siendo probablemente la menos estudiada en relación a la adicción. El tratamiento efectivo de las drogodependencias está íntimamente ligado a la evaluación y posible intervención sobre características psicológicas individuales que pueden condicionar el resultado terapéutico. Por ello, es preciso contar con instrumentos de evaluación fiables y válidos que permitan delimitar las características personales, fortalezas y debilidades, de las personas que van a realizar un tratamiento. El VIP parece ser una herramienta útil en la evaluación psicológica, en la medida en que proporciona, en una sola administración,

Correspondencia: Eduardo J. Pedrero-Pérez. Unidad de Formación e Investigación. CAD San Blas. Instituto de Adicciones. Madrid Salud.

Email: ejpedrero@yahoo.es

${ }^{1}$ Unidad de Formación e Investigación. CAD San Blas. Instituto de Adicciones. Madrid Salud, España.

Recibido: 20 de diciembre de 2015.

Aceptado: 12 de marzo de 2016. 
datos sobre un buen número de variables psicológicas, es una prueba gratuita, de libre disposición para los clínicos e investigadores y fácil corrección e interpretación.

Palabras clave: evaluación psicológica; adicción; afrontamiento; autoestima; optimismo; análisis factorial confirmatorio.

\begin{abstract}
The Questionnaire of Psychosocial Interaction Variables (VIP) was proposed a decade ago to explore psychological variables critically related with addiction: self-esteem, ogeneral self-efficacy, optimism, locus of control, social skills, self-control and coping styles. The present study was conducted a confirmatory factor analysis on a sample of 1,242 subjects treated for addiction and has studied the reliability of the scales and predictive validity on health variables and perceived stress. The factorial study showed adequate construct validity and all scales showed proper reliability, except that of locus of control, with only three items. Several scales show high correlations with perceived stress scale, quality of life and healthrelated risk of poor mental health. It stresses the predictive capacity of the scale of optimism on these three variables, probably being the least studied in relation to addiction. Effective treatment of drug addiction is closely linked to the evaluation and possible intervention on individual psychological characteristics that may influence the therapeutic outcome. Therefore, it is necessary to have reliable and valid instruments that allow the assessment to delimit the personal characteristics, strengths and weaknesses of the people who start a treatment. The VIP appears to be a useful tool in psychological assessment, as it provides, in a single administration, data on a number of psychological variables, it is a free tool, freely available to clinicians and easy correction and interpretation.
\end{abstract}

Keywords: Psychological assessment; addiction; coping; self-esteem, optimism; confirmatory factor analysis.

\section{Introducción}

La importancia de los factores psicológicos en la adicción y su recuperación está fuera de toda duda, constituyéndose en elementos centrales de la prevención y el tratamiento de rehabilitación, incluso en los casos en que se acompañan de otras modalidades terapéuticas (Gifford y Humphreys, 2007). Y existe evidencia científica de que los tratamientos psicológicos son, en general, los más efectivos (Becoña Iglesias y Cortés Tomás, 2008). Sin embargo, es necesario individualizar el ritmo de incorporación de cada sujeto, de modo que el programa se adapte a sus características y no al revés.

En este sentido, hace ahora una década se propuso un instrumento de evaluación psicológica: el Cuestionario de Variables de Interacción Psicosocial (VIP), que permite la exploración de variables como la autoestima, la autoeficacia percibida, el optimismo, el locus de control, las habilidades sociales, el autocontrol y los estilos de afrontamiento del estrés (Pedrero Pérez, Pérez López, De Ena de la Cuesta y Garrido Caballero, 2005). La baja autoestima se vincula a la adicción tanto como rasgo favorecedor del establecimiento de patrones desadaptativos de consumo, como un factor de mantenimiento de la conducta y como una consecuencia de la incapacidad percibida para mantener el control; ello no sólo se ha encontrado en adicción a sustancias, sino también en las denominadas adicciones comportamentales sin sustancia (Sariyska et al., 2014). Su combinación con un bajo optimismo está en la base del modelo cognitivo de depresión de Beck (2008). La autoeficacia general, es decir, la capacidad estimada de enfrentarse las dificultades que evalúa cada sujeto, se relaciona con su capacidad real para enfrentarse a situaciones de riesgo, así como para mantenerse en tratamiento y alcanzar buenos resultados (Kadden y Litt, 2011). El locus de control interno es la creencia de que el sujeto está en condiciones (capacidades y esfuerzo) de modificar su conducta, mientras que si es externo tenderá a modificarla poco en espera de que circunstancias externas (intervención médica/psicológica, medicación, suerte, control parental) sean la clave del cambio; es pues vital considerar el sistema de creencias del individuo a la hora de proponer un tratamiento (Ersche, Turton, Croudace y Štochl, 2012). La falta de habilidades sociales se relaciona con el desarrollo de patrones 
de consumo desadaptativos, así como con otros problemas psicopatológicos asociados a la adicción (Beardslee, Chien y Bell, 2014). El autocontrol sería la dimensión opuesta a la impulsividad, y, más precisamente, la capacidad aprendida para inhibir respuestas irreflexivas sin cálculo de consecuencias (de Ridder, Lensvelt-Mulders, Finkenauer, Stok y Baumeister, 2012). Finalmente, la manera o estilo general de resolver los conflictos es una variable crítica en relación con el abuso de sustancias: si lo deseable es una orientación al problema y su resolución, y la búsqueda de apoyo social es una segunda línea de afrontamiento, la orientación al malestar no sólo es ineficaz, sino que favorece la evitación y el escape, siendo el consumo de drogas el principal mecanismo de reducción del malestar (Corbin, Farmer y Nolen-Hoekesma, 2013). Un gran número de estudios relacionan estas variables con calidad de vida, salud mental y cualquier patología conocida.

Desde entonces, el cuestionario se ha utilizado en diversos ámbitos clínicos, y en estudios empíricos: relación de estas variables con rasgos de personalidad de diferentes modelos (Pedrero Pérez, 2006, 2007, 2008a; Pedrero Pérez, Olivar Arroyo y Puerta García, 2007) o con trastornos de la personalidad (Pedrero Pérez, López-Durán y Olivar Arroyo, 2006) variables relacionadas con el consumo de sustancias (Chicharro Romero, Pedrero Pérez y Pérez López, 2007; Pedrero Pérez, Rojo Mota y Puerta García, 2008), o adicciones comportamentales (Pedrero Pérez, 2010), su prevención (Cortés, Espejo, Dolz, Giménez y Belda, 2009) o su tratamiento (Calderón, 2008; Carreras, Almendros y Carrobles, 2015; Martínez-González, Albein-Urios, Lozano-Rojas y VerdejoGarcía, 2015; Pedrero Pérez, 2008b), o con calidad de vida (Pedrero Pérez, Olivar Arroyo y Chicharro Romero, 2008), diferencias de género (de Ena de la Cuesta y Pedrero Pérez, 2004) o valores sociales (Pedrero Pérez, Rojo Mota y Puerta García, 2009).

Sin embargo, ningún trabajo ha abordado la réplica de sus propiedades psicométricas desde que fuera propuesto en 2005. La teórica estructura de nueve escalas inicialmente planteada no se replicaba en su totalidad en el estudio inicial, agrupándose ciertas escalas entre sí, reflejando la existencia de suprafactores de carácter más general que los de la propuesta teórica. Se optó por man- tener la estructura de nueve escalas en la medida en cada una de ellas prescribe acciones concretas en el diseño del tratamiento individualizado: por ejemplo, la terapia de resolución de conflictos para el afrontamiento centrado en la tarea, las técnicas de control emocional para el afrontamiento centrado en la emoción o las técnicas de autocontrol para la escala del mismo nombre. El hecho de primar la utilidad clínica no exime del cumplimiento de adecuadas propiedades psicométricas para el instrumento de medida.

El objetivo principal del presente trabajo es explorar la validez de constructo del VIP, confirmando o rechazando la adecuación de la propuesta teórica nuevas muestras de sujetos en tratamiento por adicción. Adicionalmente, y una vez conocidas las propiedades o limitaciones del instrumento, se estudiarán diferencias entre grupos de consumidores según la droga principal o diferencias de género, así como su relación con medidas de estrés percibido, calidad de vida y salud mental.

\section{Método}

\section{Participantes}

La población objeto de estudio está constituida por los 10107 estudiantes que se han presentado a las pruebas referidas en el periodo objeto de estudio. En la Tabla 1 se muestran dichos sujetos agrupados de acuerdo con la universidad de origen.

En total se contemplan 40 universidades públicas y privadas, que ofertan la titulación de Psicología en la modalidad presencial y a distancia. El resto de universidades que imparten estos estudios en España (ocho de titularidad privada y una de titularidad pública) no han sido consideradas, ya que ningún estudiante de estas instituciones se ha presentado a las pruebas selectivas para el acceso al SNS. La muestra estuvo compuesta por 1242 personas en tratamiento por adicción a sustancias que accedían a un tratamiento por adicción a sustancias en un centro específico, público y gratuito de Madrid (CAD San Blas, 
Tabla 1

Descriptivos de la muestra

\begin{tabular}{|c|c|c|}
\hline \multirow[b]{2}{*}{ Sexo } & \multicolumn{2}{|c|}{ Muestra } \\
\hline & Varones & Mujeres \\
\hline $\mathrm{n}$ & 923 & 319 \\
\hline$\%$ & $74.30 \%$ & $25.70 \%$ \\
\hline \multicolumn{3}{|l|}{ Edad } \\
\hline Media & 36.6 & 37.4 \\
\hline D. t. & 9.2 & 10.1 \\
\hline Rango & $18-67$ & $18-66$ \\
\hline Nivel de estudios & & \\
\hline Primarios o menos & 17.4 & 15.7 \\
\hline Secundaria obligatoria & 40.2 & 30.4 \\
\hline Secundaria posobligatoria & 29.4 & 36.4 \\
\hline Universitarios & 13.0 & 17.6 \\
\hline Droga principal & & \\
\hline Heroína & 14.8 & 13.5 \\
\hline Cocaína & 41.6 & 32.6 \\
\hline Alcohol & 35.6 & 46.7 \\
\hline Cannabis & 7.9 & 7.2 \\
\hline
\end{tabular}

Instituto de Adicciones) y estuvo formada por 923 varones y 319 mujeres, en una proporción de 3/1 habitual en los centros de tratamiento a lo largo del tiempo. Los sujetos cumplimentaban una amplia batería de pruebas de evaluación, entre las que se encontraba el VIP y el resto de pruebas utilizadas en el presente estudio. Todos ellos fueron informados del doble objetivo de la evaluación (diseño individualizado del tratamiento e investigación) y firmaron un consentimiento informado. En la Tabla 1 se muestran detalladamente los descriptivos de la muestra.

\section{Instrumentos de evaluación}

Cuestionario de Variables de Interacción Psicosocial (VIP; Pedrero Pérez et al., 2005), prueba de 84 ítems, que se responden en una escala de cuatro posiciones: Muy de acuerdo, De acuerdo, En desacuerdo y Muy en desacuerdo. Las opciones se corrigen 2, 1, -1 y -2 , siempre en dirección al constructo explorado en cada escala, de modo que deben invertirse las puntuaciones de los ítems negativos. Las escalas se puntúan entre el máximo posible $(+100)$ y el mínimo posible $(-100)$ para cada escala, según la fórmula:

\section{(Puntuación directa * 100)}

$2 *$ (Ítems respondidos)

Cuando una respuesta se encuentra en blanco (o cuando el sujeto responde marcando entre las dos opciones centrales) se corrige como 0 . Se considera válido cuando no hay más de cinco respuestas en blanco. Las escalas teóricamente propuestas son: Autoestima, la Autoeficacia Percibida, el Optimismo, el Locus de Control, las Habilidades Sociales, el Autocontrol y los Estilos de Afrontamiento del Estrés. En la Tabla 2 se hacen constar los ítems que componen cada escala.

Adicionalmente, se administraron a muestras menores los siguientes cuestionarios:

Escala de Estrés Percibido de 10 items, EEP (Perceived Stress Scale, PSS; Cohen, Kamarck y Mermelstein, 1983), versión española de Remor y Carrobles (2001). La EEP está formada por 14 ítems que miden el grado en que, durante el último mes, las personas se han sentido molestas o preocupadas o, por el contrario, se han sentido seguras de su capacidad para 
Tabla 2

Ítems del VIP

\begin{tabular}{lrlr}
\hline & $\mathbf{n}^{\mathbf{0}}$ & \multicolumn{1}{l}{ ítems } & $\omega$ \\
\hline Autoestima & 12 & $(8),(13),(20),(26),(27),(37),(45),(49), 54,61,(63)$, & .92 \\
Autoeficacia & 9 & $4,6,12,23,29,40,53,57,73$ & .86 \\
Optimismo & 11 & $(3),(7),(10),(34),(42), 47,(51), 66,68,(69),(75)$ & .86 \\
Locus (internalidad) & 3 & $(32),(39),(81)$ & .61 \\
Habilidades sociales & 10 & $(2),(11),(18),(30),(33),(35),(38), 59,(71),(79)$ & .84 \\
Autocontrol & 11 & $(5), 16,17,19,22,(25),(50), 56,(78),(83),(84)$ & .82 \\
Afrontamiento centrado en la tarea & 11 & $9,15,24,28,31,44,48,52,58,60,76$ & .90 \\
Afrontamiento centrado en la emoción & 12 & $1,21,36,43,46,55,62,65,72,74,77,82$ & .87 \\
Afrontamiento centrado la búsqueda de apoyo & 5 & $14,41,64,67,80$ & .82 \\
social & 5 & & \\
\hline
\end{tabular}

Nota. Entre paréntesis, los ítems que deben invertirse. $\omega=$ Omega de McDonald.

controlar sus problemas personales. La escala puntúa entre $0-40$, con puntaciones superiores indicando mayor estrés percibido. Utiliza un formato de respuesta de tipo Likert de cinco alternativas con un rango de 0 («Nunca») a 4 («Muy a menudo»). Aunque la escala completa consta de 14 ítems, se ha encontrado que en población clínica y no clínica española (aunque también en muestras de otras procedencias e idiomas) la versión de 10 ítems presenta los mejores indicadores psicométricos (Pedrero-Pérez et al., 2015)

El COOP/WONCA (Skevington, Sartorius, Amir y THE WHOQOL Group) es un instrumento propuesto por la Organización Mundial de Médicos de Familia (WONCA) para estimar la Calidad de Vida Relacionada con la Salud. Se utilizó la versión española de Lizan y Reig (1999). Los ítems exploran aspectos relativos a esta variable a través de unas láminas en las que se visualizan mediante dibujos las 5 opciones de respuestas, debiendo marcar el evaluado la que mejor defina su estado. Aunque se han utilizado versiones de seis, siete y nueve ítems, para el presente estudio se optó por la versión más amplia, de nueve ítems, baremada en población española (Serrano-Gallardo, Lizán-Tudela y Díaz-Olalla, 2009) y sin hacer uso de los estímulos visuales, sino sólo los contenidos de cada ítem como estímulos verbales, lo que ha mostrado no presentar ni diferencias psicométricas significativas ni ventajas percibidas por el cumplimentador, pudiendo administrarse incluso mediante entrevista telefónica (Pedrero-Pérez y Díaz-
Olalla, 2014). Las respuestas corresponden a una escala tipo Likert, en la que mayores puntuaciones se corresponden con peor calidad de vida percibida.

Cuestionario General de Salud de 12 items, GHQ-12 (The 12-Item General Health Questionnaire, GHQ-12; Goldberg y Williams, 1988), instrumento para cribar posibles problemas de salud mental (salvo los psicóticos). Se responde en una escala Likert de 4 opciones (0 nada, 3 siempre; se invierten los ítems negativos), correspondiendo mayores puntuaciones a más probable existencia de problemas de salud mental. Se corrige de dos maneras complementarias: las dos primeras opciones como 0 y las dos siguientes como 1; se obtiene una puntuación entre 0 y 12 puntos, considerándose que tres o más sugieren "riesgo de mala salud mental"; la segunda forma de corrección es como una escala tipo Likert normal, con puntuaciones entre 0 y 36 , lo que da idea de la intensidad del riesgo. Ha mostrado adecuadas propiedades psicométricas en su versión española (Sánchez-López y Dresch, 2008).

\section{Procedimiento}

Se procedió a un muestreo consecutivo de las personas admitidas a tratamiento en el Centro, entre enero de 2010 y junio de 2015, que realizaron la prueba cuando estuvieron libres de intoxicación por sustancias 
no prescritas, esperando para ello entre dos y cuatro semanas en la mayor parte de los casos, y comprobándose mediante análisis toxicológicos de orina $\mathrm{o}$ análisis de aire espirado. Los participantes eran advertidos del propósito de la prueba y accedieron a cumplimentarla de manera anónima y desinteresada. Se excluyeron los cuestionarios deficientemente cumplimentados $(n=16)$. Para su inclusión, los sujetos debían cumplir criterios de abuso o dependencia de al menos una sustancia al inicio de su tratamiento y según criterios DSM-IV. Se excluyó a quienes carecían de un adecuado nivel de comprensión del idioma español.

\section{Análisis de datos}

Se analizaron en primer lugar los 84 ítems, explorando sus distribuciones. Para la determinación de la normalidad multivariada se utilizó el criterio de Mardia (1970). Se realizó un análisis factorial confirmatorio para explorar la estructura de nueve escalas propuestas teóricamente; se utilizó el método de mínimos cuadrados no ponderados, como el más adecuado a las opciones de respuesta (categorial, no lineal). Se utilizaron indicadores de bondad de ajuste generales (CMIN/DF), basados en los residuos (RMSEA) y los proporcionados por el programa AMOS 18.0 (RMR, GFI, AGFI, NFI, RFI, PNFI y PGFI). La consistencia interna de las escalas se estimó mediante el omega de McDonald (1999). Se estudio el efecto de interacción de las variables mediante la lambda de Wilks $(\lambda)$. Se calcularon las correlaciones parciales, controlando las variables con efecto de interacción, y las de orden cero, utilizando la corrección de Bonferroni para correlaciones múltiples, con objeto de eliminar las correlaciones al azar. Para las comparaciones entre subgrupos se aplicó un análisis de covarianza. Las relaciones predictivas entre variables se estudiaron a partir de los pesos de regresión (índice de regresión dividido por el error estándar). El tamaño del efecto se calculó mediante la eta al cuadrado $\eta^{2}$; Cohen, 1973), aplicando las reglas de interpretación propuestas por Cohen (1988).

\section{Resultados}

En primer lugar se exploró la distribución de los ítems para descartar la existencia de outliers, esto es, ítems con asimetría mayor de tres o curtosis superior a ocho. La asimetría se mantuvo en valores entre -1.23 y 0.88 , mientras que la curtosis osciló entre -1.56 y 1.07. En su conjunto, los datos cumplieron el criterio de normalidad multivariada (Mardia 153.1, $p .0001$ ).

También cumplían las condiciones para realizar un análisis factorial $($ Bartlett $=36482$, g.l. $=3486, p<.0001$; Kaiser-Meyer-Olkin $=.96$ ). Las escalas explicaban en su conjunto el $47 \%$ de la varianza total del test.

Se procedió a efectuar un análisis factorial confirmatorio para explorar el ajuste del modelo teórico de nueve escalas a los datos disponibles. El índice de bondad de ajuste general resultó satisfactorio $\chi^{2}=10363.8$, g.l. $\left.=3366, p<.0001, \mathrm{CMIN} / \mathrm{DF}=3.0\right) \mathrm{y}$ también indicadores no sensibles al tamaño de la muestra $($ RMSEA $=.045)$. Cuando se efectuó un análisis de mínimos cuadrados no ponderados, todos los indicadores mostraron una adecuada bondad de ajuste $(\mathrm{RMR}=.08$, $\mathrm{GFI}=.96, \mathrm{AGFI}=.96, \mathrm{NFI}=.95, \mathrm{RFI}=.95, \mathrm{PNFI}=.92$, PGFI $=.90$ ). Por tanto, se confirmó como adecuada la estructura de nueve escalas del VIP. Todas las escalas mostraron adecuada consistencia interna, salvo la de locus de control, compuesta por sólo tres ítems (Tabla 2).

Se compararon las puntuaciones obtenidas por los grupos, según la droga principal que motivaba su tratamiento. Previamente, se exploró el posible efecto de interacción de diversas variables, que podía afectar a las diferencias. Mostraron efecto de interacción el sexo $(\lambda$ de Wilks $\left.=.94, \mathrm{~F}_{(9.0 ; 1227,0)}=8.36, p<.001, \eta^{2=} .06\right)$, la edad $\left(\lambda=.95, \mathrm{~F}_{(\mathrm{g} .1 .9,0 ; 1227,0)}=7.76, p<.001, \eta^{2=} .05\right)$ y el nivel de estudios $\left(\lambda=.96, \mathrm{~F}_{(9.0 ; 1227.0)}=5.82, p<.001\right.$, $\left.\eta^{2}=.04\right)$. Por ello, estas tres variables fueron controladas en análisis posteriores.

En la Tabla 3 se muestran las correlaciones entre las escalas del VIP, tanto cuando se controlan las variables en interacción, como las de orden 0 . Se observan algunas 
Tabla 3

\section{Correlaciones entre las escalas del VIP}

\begin{tabular}{|c|c|c|c|c|c|c|c|c|c|}
\hline & 1 & 2 & 3 & 4 & 5 & 6 & 7 & 8 & 9 \\
\hline 1. Autoestima & & .59 & .75 & .46 & .52 & .54 & .54 & -.69 & .13 \\
\hline 2. Autoeficacia & .60 & & .54 & .34 & .42 & .46 & .73 & -.49 & .14 \\
\hline 3. Optimismo & .76 & .55 & & .49 & .42 & .46 & .49 & -.6 & .12 \\
\hline 4. Locus (internalidad) & .47 & .35 & .50 & & .36 & .31 & .30 & -.44 & .01 \\
\hline 5. Habilidades sociales & .52 & .42 & .43 & .37 & & .22 & .29 & -.43 & .15 \\
\hline 6. Autocontrol & .54 & .47 & .46 & .32 & .21 & & .53 & -.58 & .09 \\
\hline 7. Afr. Centr. en la tarea & .55 & .74 & .49 & .31 & .29 & .54 & & -.47 & .22 \\
\hline 8. Afr. Centr. en la emoción & -.70 & -.51 & -.61 & -.45 & -.43 & -.58 & -.49 & & .02 \\
\hline 9. Afr. Centr. en apoyo social & .11 & .12 & .11 & .00 & .16 & .05 & .19 & .05 & \\
\hline
\end{tabular}

Nota. En la parte superior y cursiva, correlaciones parciales, controlando sexo, edad y nivel de estudios. En la parte inferior, correlaciones de orden 0 .

correlaciones muy elevadas y con gran tamaño del efecto: la autoestima y el optimismo $\left(r^{2}=.56\right)$ y la autoeficacia percibida y el afrontamiento centrado en la tarea $\left(r^{2}=.54\right)$. El afrontamiento centrado en la emoción correlaciona negativamente con todas las demás, especialmente con la autoestima $\left(r^{2}=.49\right)$, mientras que el afrontamiento centrado en la búsqueda de apoyo social muestra correlaciones muy bajas con el resto de las escalas.
En la Tabla 4 se muestran las diferencias encontradas en los subgrupos según la droga principal por la que se solicitó tratamiento. Puede observarse que, por lo general, las peores puntuaciones (menores en variables positivas, como la autoestima, autoeficacia, optimismo, etc.; y mayores en afrontamiento centrado en la emoción) correspondieron a los que tenían el alcohol como droga principal. En todo caso, el tamaño de las diferencias se situó entre bajo y moderado en todos los casos.

Tabla 4

Diferencias en las escalas del VIP según droga principal

\begin{tabular}{|c|c|c|c|c|c|c|c|c|c|c|}
\hline & \multicolumn{8}{|c|}{ Droga principal } & \multirow[b]{3}{*}{$F(g . I=6)$} & \multirow[b]{3}{*}{$\eta^{2}$} \\
\hline & \multicolumn{2}{|c|}{$\begin{array}{c}\text { HEROÍNA } \\
n=180\end{array}$} & \multicolumn{2}{|c|}{$\begin{array}{l}\text { COCAÍNA } \\
n=488\end{array}$} & \multicolumn{2}{|c|}{$\begin{array}{c}\text { ALCOHOL } \\
n=478\end{array}$} & \multicolumn{2}{|c|}{$\begin{array}{c}\text { CANNABIS } \\
n=96\end{array}$} & & \\
\hline & $\mathrm{M}$ & D.T. & M & D.T. & $\mathrm{M}$ & D.T. & $\mathrm{M}$ & D.T. & & \\
\hline Autoestima & 11.46 & 42.95 & 12.01 & 46.00 & 7.57 & 44.95 & 15.50 & 47.82 & 6.30 & .03 \\
\hline Autoeficacia & 16.89 & 35.81 & 18.34 & 39.58 & 12.73 & 37.01 & 16.60 & 39.16 & 6.73 & .03 \\
\hline Optimismo & 7.69 & 35.40 & 10.78 & 38.75 & 3.19 & 36.99 & 5.67 & 40.18 & 6.43 & .03 \\
\hline Locus (internalidad) & 33.68 & 46.12 & 28.32 & 47.46 & 23.42 & 46.97 & 18.40 & 46.62 & 9.47 & .04 \\
\hline Habilidades sociales & 16.22 & 38.96 & 19.28 & 38.46 & 8.52 & 41.42 & 18.18 & 38.84 & 7.57 & .04 \\
\hline Autocontrol & 2.87 & 31.58 & -0.76 & 35.95 & 4.26 & 32.19 & 1.96 & 36.64 & 8.68 & .04 \\
\hline $\begin{array}{l}\text { Afrontamiento centrado en } \\
\text { la tarea }\end{array}$ & 16.64 & 37.24 & 14.27 & 40.95 & 18.67 & 37.81 & 20.35 & 35.22 & 6.39 & .03 \\
\hline $\begin{array}{l}\text { Afrontamiento centrado en } \\
\text { la emoción }\end{array}$ & 0.14 & 37.64 & 1.53 & 38.76 & 7.76 & 37.66 & 3.53 & 39.10 & 11.21 & .05 \\
\hline $\begin{array}{l}\text { Afrontamiento centrado en } \\
\text { la búsqueda de apoyo social }\end{array}$ & -11.33 & 39.82 & -0.08 & 43.44 & -3.31 & 43.20 & 4.85 & 43.39 & 9.01 & .04 \\
\hline
\end{tabular}

Nota. $\eta^{2}=$ eta al cuadrado como estimador del tamaño del efecto. Todas las diferencias son significativas para $p<.001$. 
Tabla 5

Diferencias por sexo en las escalas del VIP

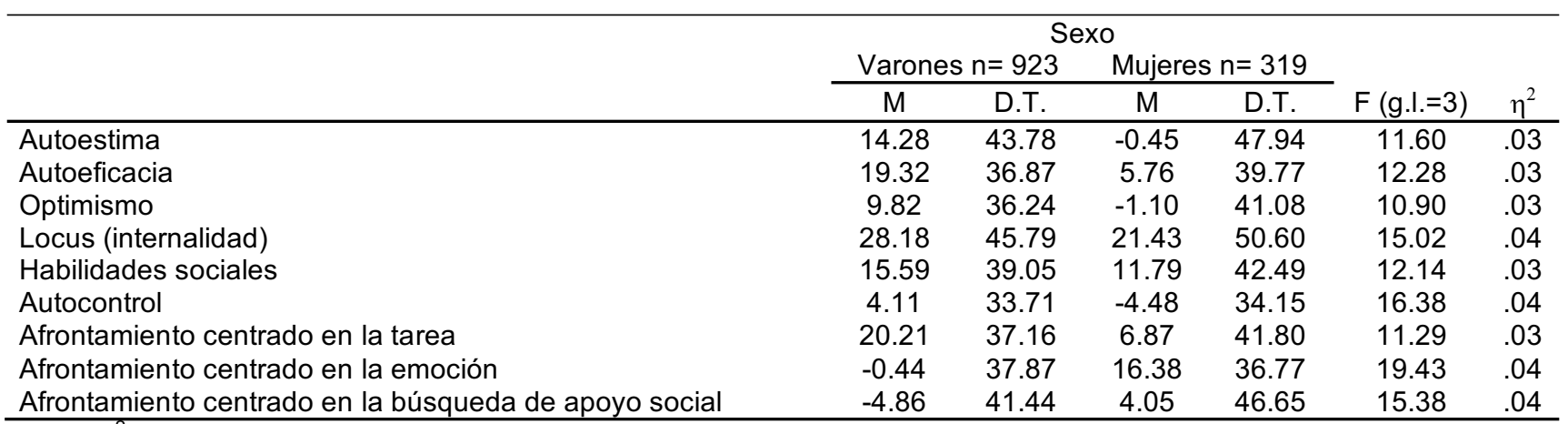

Nota. $\eta^{2}=$ eta al cuadrado como estimador del tamaño del efecto. Todas las diferencias son significativas para $p<.001$.

Las mujeres obtuvieron puntuaciones más bajas que los varones en todas las escalas, salvo en dos estilos de afrontamiento: centrado en la emoción y centrado en la búsqueda de apoyo social (Tabla 5). Estas diferencias fueron significativas, aunque el tamaño del efecto fue entre bajo y moderado. Cuando se estudiaron las diferencias por sexo en cada uno de los grupos según la droga principal, estos resultados tendían a repetirse en todas las submuestras, alcanzando un tamaño del efecto grande $\left(\eta^{2}=.07\right)$ en las dos escalas mencionadas. Sin embargo, en los consumidores de cannabis aparecían atenuados $\left(\eta^{2}=.05\right)$ y sin significación estadística en ningún caso, aunque el grupo de mujeres consumidoras de cannabis era demasiado bajo para obtener conclusiones sólidas $(n=23)$.

La edad mostró correlación significativa y positiva con el autocontrol $(r=.15, p<.001)$ y negativa con las habilidades sociales $(r=-.11, p<.001)$ y con el afrontamiento centrado en la búsqueda de apoyo social $(r=-$ $.16, p<.001)$, pero no con el resto de escalas. Estas correlaciones se mantuvieron cuando se controlaron las variables sexo y nivel de estudios.

En cuanto al nivel de estudios, mostró correlaciones significativas $(p<.05)$ con varias escalas, pero tras aplicar la corrección de Bonferroni sólo resultaron significativas en el caso del optimismo $(r=.08, p<.005)$, el locus de control interno $(r=.17, p<.001)$ y las habilidades sociales $(r=.12, p<.001)$.

Finalmente, en la Tabla 6 se observan las correlaciones encontradas entre el VIP y los autoinformes de estrés percibido, calidad de vida relacionada con la salud y riesgo de mala salud mental. Se observa que, salvo la escala de afrontamiento centrado la búsqueda de apoyo social, todas las demás muestran correlaciones ne-

Tabla 6

Correlación entre escalas del VIP y otros autoinformes

\begin{tabular}{lccc}
\hline & EEP-10 & WONCA & GHQ-12 \\
& $\mathrm{n}=552$ & $\mathrm{n}=50$ & $-.51^{*}$ \\
\hline Autoestima & $-.58^{*}$ & $-.55^{*}$ & $-.49^{*}$ \\
Autoeficacia & $-.50^{*}$ & $-.57^{*}$ & $-.57^{*}$ \\
Optimismo & $-.57^{*}$ & $-.64^{*}$ & -.30 \\
Locus (internalidad) & $-.32^{*}$ & -.38 & -.10 \\
Habilidades sociales & $-.29^{*}$ & -.24 & -.27 \\
Autocontrol & $-.49^{*}$ & -.35 & $-.44^{*}$ \\
Afrontamiento centrado en la tarea & $-.46^{*}$ & $-.44^{*}$ & $.44^{*}$ \\
Afrontamiento centrado en la emoción & $.58^{*}$ & $.44^{*}$ & .04 \\
Afrontamiento centrado la búsqueda de apoyo social & -.03 & -.02 & \\
\hline
\end{tabular}

Nota. ${ }^{*}$ Significativo tras corrección de Bonferroni $(p<.006)$. 


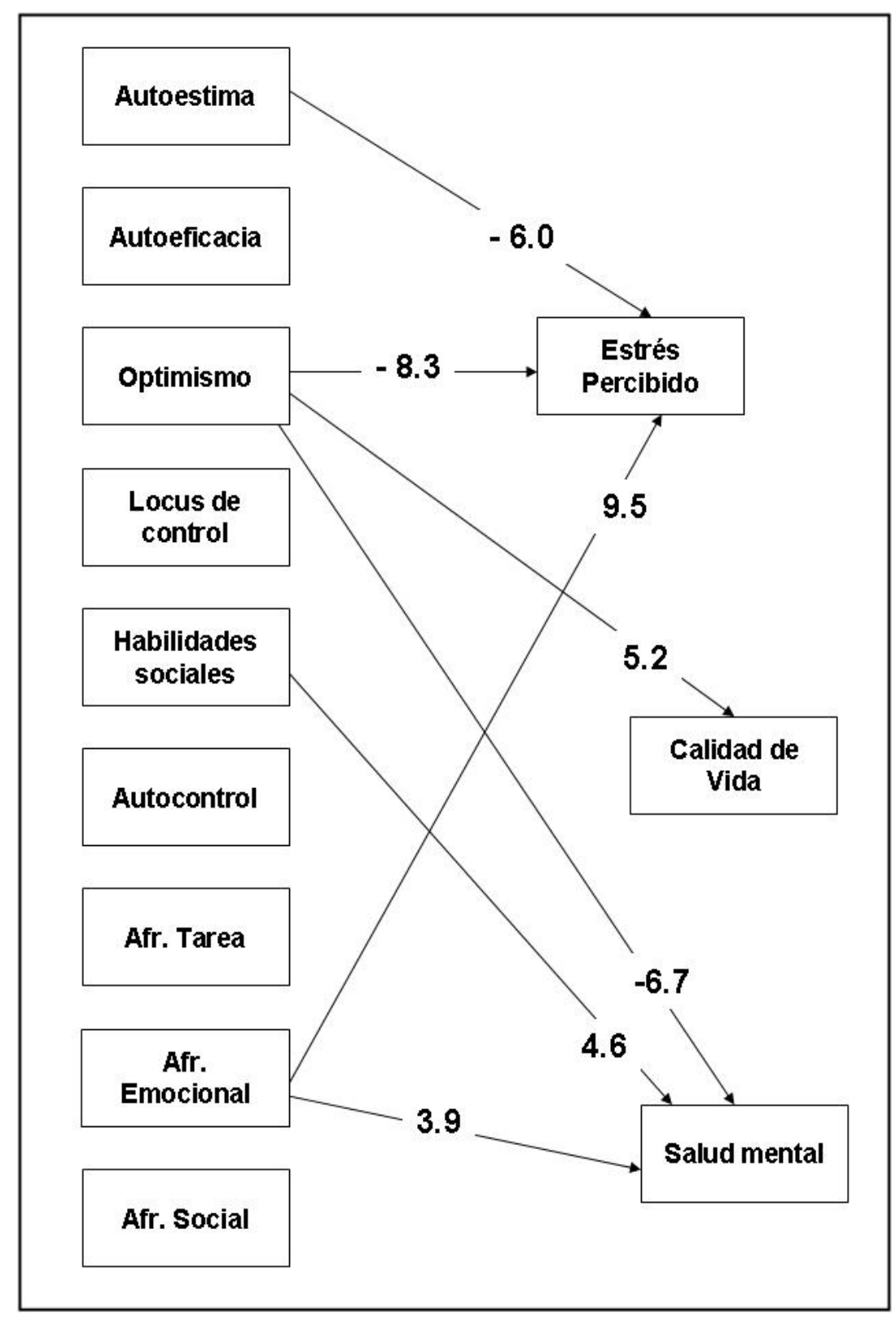

Figura 1. Relación entre las variables (pesos de regresión).

gativas y, en su mayor parte, significativas con las variables estudiadas. Los resultados son muy similares cuando se efectúa el análisis correlacional por sexos. En la Figura 1 se muestran las relaciones predictivas entre las escalas del VIP y el resto de autoinformes. 


\section{Discusión}

El tratamiento efectivo de las drogodependencias está íntimamente ligado a la evaluación y posible intervención sobre las características psicológicas individuales que pueden condicionar el resultado terapéutico (Becoña Iglesias y Cortés Tomás, 2008). Por ello, es preciso contar con instrumentos de evaluación fiables y válidos que permitan delimitar las características personales, fortalezas y debilidades, de las personas que van a realizar un tratamiento.

El VIP fue concebido como un instrumento que permitiera la evaluación de ciertas variables críticas en la estructura de personalidad de las personas con conductas adictivas. Su ventaja es que con una sola administración pueden evaluarse hasta nueve de estas variables, desde la autoestima hasta los estilos de afrontamiento del estrés.

La estructura de nueve escalas ha sido confirmada mediante análisis factorial confirmatorio, resultando satisfactorios todos los indicadores de bondad de ajuste. Es posible que la agrupación de lagunas escalas proporcionara soluciones más parsimoniosas, en la medida en que algunos constructos, medidos en diferentes escalas, se encuentran conceptualmente muy próximos. Por ejemplo, la autoeficacia percibida parece un prerrequisito para el afrontamiento de las dificultades orientado a su resolución. Sin embargo, parece de interés para la clínica distinguir entre ambas variables, en la medida en que pueden ser trabajadas de maneras diferentes, aunque complementarias. El uso del VIP permite el diseño de un menú personalizado de intervenciones psicoterapéuticas sobre aspectos básicos relacionados con la conducta adictiva.

Todas las escalas se han mostrado consistentes salvo la de locus de control, compuesta sólo de 3 ítems. Conservar esta escala puede ser importante porque nos informa de las creencias que guían al sujeto al solicitar tratamiento. El modelo de enfermedad de la adicción ha favorecido que muchas personas crean tener una patología en cuya solución tienen un mínimo papel.
Esperarían, pues, que fuera la intervención externa la que resolviera sus problemas, lo que no sólo tiene poca relación con la realidad, sino que es uno de los principales factores de recaída y abandono del tratamiento (Miller, Westerberg, Harris y Tonigan, 1996). Es preciso conocer el sistema de creencias y modificarlo en dirección a una participación activa del sujeto en el proceso de cambio.

Cuando se observan las puntuaciones obtenidas por los participantes y se comparan con las obtenidas en la muestra inicial de validación. La diferencia crucial entre ambos estudios estriba en que en aquella ocasión el VIP fue administrado a sujetos que se encontraban en muy diversas fases de tratamiento, mientras que en el presente estudio la muestra ha estado compuesta sólo por personas que iniciaban su tratamiento. Es conocido que los síntomas de malestar psicológico son máximos en el inicio del tratamiento, siendo, en realidad, la principal motivación para solicitarlo (Pedrero Pérez, Puerta García, Segura López y Osorio del Río, 2004). Este malestar, provocado por las consecuencias del consumo (estrés psicosocial, restricción estimular, efectos de las sustancias), tiende a desaparecer en los meses siguientes, incluso en el caso de las supuestas patologías estables, como el caso de los trastornos de la personalidad (Pedrero-Pérez, López-Durán y OlivarArroyo, 2011). Diseñar programas individualizados puede acelerar esta desaparición y mejorar los resultados del tratamiento.

La comparación según la droga principal aporta sutiles diferencias, que se hacen más acentuadas en el caso de consumidores de alcohol. Los consumidores problemáticos de esta sustancia tardan más en solicitar tratamiento, presentan mayor edad al hacerlo, su consumo es más fácilmente aceptado por su entorno y con frecuencia presentan mayor deterioro debido, entre otras causas, a déficits en la alimentación (Pfefferbaum, Adalsteinsson y Sullivan, 2006). Las mujeres obtienen peores puntuaciones, como suele ser normal en casi todos los autoinformes de salud física y mental, sin que exista certeza de que ello se debe a problemas biológicos (e.g., hormonales), psicológicos (e.g., historial de aprendizaje diferencial) o de otra índole (Kramer, Krueger y Hicks, 2008). En todo caso, se 
requieren baremos diferenciados para hombres $\mathrm{y}$ mujeres en la correcta interpretación de ésta y otras pruebas.

La importancia de estas variables queda firmemente sustentada en el hecho de que estas variables muestran correlaciones de gran magnitud con otras referidas a la calidad de vida y el riesgo de desarrollar problemas de salud mental. Cuando se estudia la capacidad predictiva de las escalas del VIP sobre estar variables externas llama la atención que la que presenta valores más elevados en todos los casos es el optimismo. No está clara la naturaleza de esta variable, que para unos es un sesgo cognitivo, para otros, una actitud disposicional o una creencia y para otros se trata de un juicio probabilístico sobre la ocurrencia de acontecimientos positivos y negativos en el futuro. Resultados metaanalíticos encuentran que los optimistas son más capaces de adaptar sus estrategias de afrontamiento a las necesidades impuestas por el estresor, obteniendo mejores resultados (Nes y Segerstrom, 2006) y es un potente predictor de buenos resultados de salud (Rasmussen, Scheier y Greenhouse, 2009). Probablemente esta sea la variable menos estudiada en el campo de la adicción, aunque puede estar relacionada con otras bien conocidas, como la resiliencia. La otra variable con gran capacidad predictiva es el afrontamiento centrado en la emoción, que se relaciona con altos niveles de estrés percibido, reflejando, probablemente, su inadecuación para la resolución de conflictos.

El presente trabajo presenta limitaciones. Por una parte, el muestreo consecutivo no proporciona resultados que puedan generalizarse. Sin embargo, el objetivo de este trabajo no era el establecimiento de baremos, sino el estudio psicométrico de un cuestionario. En este sentido, se exige un mínimo de 10 sujetos por ítem, algo que se ha superado ampliamente en este trabajo, alcanzando una relación de $15 / 1$, por lo que el estudio factorial debe considerarse adecuado y sus resultados estables. En segundo lugar, de las muestras que han cumplimentado algunos cuestionarios complementarios son excesivamente pequeñas, lo que sugiere estudios con muestras más amplias para dar valor a los resultados obtenidos. Finalmente, se carece de estudios en poblaciones no clínicas que permitan obtener baremos e interpretar adecuadamente los alcanzados por la muestra clínica en tratamiento por adicción.

En definitiva, el VIP parece ser una herramienta útil en la evaluación psicológica, en la medida en que proporciona, en una sola administración, datos sobre un buen número de variables psicológicas críticas relacionadas con la adquisición, el mantenimiento y el abandono de la adicción. Su uso puede permitir el diseño individualizado de programas de tratamiento enfocados a las variables más relacionadas con la salud física $\mathrm{y}$ mental de quienes inician un tratamiento y puede ser utilizada como medida de cambio tras el tratamiento. Esta prueba ha mostrado adecuada validez de constructo, fiabilidad en sus escalas y validez predictiva sobre indicadores de salud. Es, por otra parte, una prueba gratuita, de libre disposición para los clínicos y fácil corrección e interpretación. 


\section{Referencias}

Beck, A. T. (2008). The Evolution of the Cognitive Model of Depression and its Neurobiological Correlates. American Journal of Psychiatry, 165, 969-977.

http://dx.doi.org/10.1176/appi.ajp.2008.08050721

Becoña Iglesias, E. y Cortés Tomás, M. T. (Eds.). (2008). Guía clínica de intervención psicológica en adicciones. Guías clínicas socidrogalcohol basadas en la evidencia científica. Barcelona, España: Socodrogalcohol.

Beardslee, W. R., Chien, P. L. y Bell, C. C. (2014). Prevention of Mental Disorders, Substance Abuse, and Problem Behaviors: A Developmental Perspective. Psychiatric Services, 62(3), 247-254 http://dx.doi.org/10.1176/appi.ps.62.3.247.

Calderón, D. (2008). Programa para mejorar las habilidades psicosociales con técnicas creativas. Intervención para jóvenes en tratamiento por drogadependencia (Tesis Doctoral). Recuperado de https://rdu.unc.edu.ar/bitstream/handle/11086/234/ Dr._Calderon_Diana.pdf? sequence $=1$.

Carreras, A., Almendros, C. y Carrobles, J. A. (2015). Variables predictoras del tratamiento de retirada de metadona en pacientes ambulatorios [Predictors of Methadone Reduction in Outpatients]. Psicología Conductual, 23(1), 85-106.

Chicharro Romero, J., Pedrero Pérez, E. J. y Pérez López, M. (2007). Autoeficacia para resistirse al consumo de sustancias como predictora de resultados de tratamiento y su relación con variables de personalidad: estudio de una muestra de adictos con el DTCQ, el VIP y el MCMI-II. Adicciones, 19(2), 141-151. Recuperado de http://www.adicciones.es/index.php/adicciones/artic le/view/312

Cohen, J. (1973). Eta-squared and Partial Eta-Squared in fixed factor ANOVA Designs. Educational and
Psychological Measurement, 33, 107-112. http://dx.doi.org/10.1177/001316447303300111

Cohen, S., Kamarck, T. y Mermelstein, R. (1983). A Global Measure of Perceived Stress. Journal of Health and Social Behavior, 24, 385-396.

Cohen, J. (1988). Statistical Power Analysis for the Behavioral Sciences (2 ${ }^{\text {a }}$ Ed.). Hillsdale, NJ: Erlbaum.

Corbin, W. R., Farmer, N. M. y Nolen-Hoekesma, S. (2013). Relations Among Stress, Coping Strategies, Coping Motives, Alcohol Consumption, and Related Problems: A Mediated Moderation Model. Addictive Behaviors, 38(4), 1912-1919. http://dx.doi.org/10.1016/j.addbeh.2012.12.005

Cortés, M. T., Espejo, B., Dolz, L., Giménez, J. A. y Belda, L. (2009). Desarrollo de un programa de prevención selectiva dirigido a jóvenes menores de 25 años sancionados por tenencia-consumo de drogas en la vía pública. Programa Mira'T. Valencia, España: Universitat de València.

De Ena de la Cuesta, S. y Pedrero Pérez, E. J. (2004). Diferencias de género en personalidad en consumidores de drogas en tratamiento: rasgos, trastornos y variables interaccionales ise justifican tratamientos diferentes? Libro de Ponencias y Comunicaciones. Encuentro de Profesionales en Drogodependencias y Adicciones. Género y Adicciones (pp. 209-223). Chiclana de la Frontera, España: Diputación de Cádiz.

De Ridder, D. T., Lensvelt-Mulders, G., Finkenauer, C., Stok, F. M. y Baumeister, R. F. (2012). Taking Stock of Self-Control. A Meta-Analysis of How Trait Self-Control Relates to a Wide Range of Behaviors. Personality and Social Psychology Review, 16(1), 76-99. http://dx.doi.org/10.1177/1088868311418749

Ersche, K. D., Turton, A. J., Croudace, T. y Štochl, J. (2012). Who do you Think is in Control in Addiction? A Pilot Study on Drug-Related Locus of 
Control Beliefs. Addictive Disorders \& Their Treatment, 11(4), 173-223. http://dx.doi.org/10.1097/ADT.0b013e31823da151

Gifford, E. y Humphreys, K. (2007). The Psychological Science of Addiction. Addiction, 102(3), 352-361. http://dx.doi.org/10.1111/j.1360-0443.2006.01706.x

Goldberg, D. y Williams, P. (1988). A User's Guide to the General Health Questionnaire. Windsor, UK: NFER-Nelson.

Kadden, R. M. y Litt, M. D. (2011). The Role of SelfEfficacy in the Treatment of Substance Use Disorders. Addictive Behaviors, 36(12), 1120-1126. http://dx.doi.org/10.1016/j.addbeh.2011.07.032

Kramer, M. D., Krueger, R. F. y Hicks, B. M. (2008). The Role of Internalizing and Externalizing Liability Factors in Accounting for Gender Differences in the Prevalence of Common Psychopathological Syndromes. Psychological Medicine, 38, 51-61. http://dx.doi.org/10.1017/S0033291707001572

Lizán L. y Reig A. (1999). Adaptación transcultural de una medida de la calidad de vida relacionada con la salud: la versión española de las viñetas COOP/WONCA [Cross-cultural Adaptation of a Health-Related Quality of Life Measurement: The SPANISH VERSION of the COOP/WONCA Cartoons]. Atención Primaria, 24, 75-82.

Mardia, K. V. (1970). Measures of Multivariate Skewnees and Kurtosis with Applications. Biometrika, 57, 519-530. http://dx.doi.org/10.2307/2334770

McDonald, R. P. (1999). Test Theory: A Unified Approach. Mahwah, NJ: Lawrence Erlbaum Associates.

Martínez-González, J. M., Albein-Urios, N., LozanoRojas, O. y Verdejo-García, A. (2015). Variables de interés clínico en el tratamiento cognitivoconductual de la adicción a la cocaína:
Especificidad de los trastornos de la personalidad [Variables of Clinical Interest in the Cognitive Behavioral Treatment of Cocaine Addiction: The Specificity of Personality Disorders]. Revista de Psicopatología y Psicología Clínica, 20(2), 115123.

http://dx.doi.org/10.5944/rppc.vol.20.num.2.2015.15 166

Miller, W. R., Westerberg, V. S., Harris, R. J. y Tonigan, J. S. (1996). What Predicts Relapse? Prospective Testing of Antecedent Models. Addiction, 91(Suppl.1), S155-S172. http://dx.doi.org/10.1046/j.1360-0443.91.12s1.7.x

Nes, L. S. y Segerstrom, S. C. (2006). Dispositional Optimism and Coping: A Meta-Analytic Review. Personality and Social Psychology Review, 10(3), 235-251.

http://dx.doi.org/10.1207/s15327957pspr1003_3

Pedrero Pérez, E. J. (2006). TCI-140: propiedades psicométricas, relación con el TCI-R y con variables de personalidad. Estudio de una muestra de adictos en tratamiento [TCI-140: Psychometric Properties, Relation with TCI-R, and Personality Variables. Studying a Sample of Addicts in Treatment]. Trastornos Adictivos, 8(3), 155-167. http://dx.doi.org/10.1016/S1575-0973(06)75116-9

Pedrero Pérez, E. J. (2007). El modelo de cinco factores y la estructura dimensional de los trastornos de la personalidad: estudio de una muestra de adictos a sustancias con el BFQ y el MCMI-II [The Model of Five Factors and Dimensional Structure of Personality Disorders: Study of a Sample of Substance Addicts with BFQ and MCMI-II]. Trastornos Adictivos, 9(2), 116-125. http://dx.doi.org/10.1016/S1575-0973(07)75637-4

Pedrero Pérez, E. J. (2008a). Personalidad y adicciones: relaciones empíricas desde diversos modelos teóricos e implicaciones para el tratamiento. Revista Peruana de Drogodependencias, 6(1), 9-54. 
Pedrero Pérez, E. J. (2008b). El tratamiento de la dependencia de la cocaína «guiado por la personalidad» [Personality Guided Treatment for Cocainedependence]. Trastornos Adictivos, 10(4), 226-241. http://dx.doi.org/10.1016/S15750973(08)76372-4

Pedrero Pérez, E. J. (2010). Detección de adicciones comportamentales en adictos a sustancias en tratamiento [Screening of Behavioral Addictions in Substance-Addicted Patient]. Trastornos Adictivos, 12(1), 13-18. http://dx.doi.org/10.1016/S15750973(10)70005-2

Pedrero-Pérez, E. J. y Díaz-Olalla, J. M. (2014). COOP/WONCA: fiabilidad y validez de la prueba administrada telefónicamente [COOP/WONCA: Reliability and Validity of the Test Administered by Telephone]. Atención Primaria, avance online. http://dx.doi.org/10.1016/j.aprim.2014.12.010

Pedrero Pérez, E. J., López-Durán, A. y Olivar Arroyo, A. (2006). El trastorno negativista de la personalidad y su relación con el abuso de sustancias [Negativistic Personality Disorder Related with Substance Abuse]. Trastornos Adictivos, $\quad 8(1), \quad 22-41$. http://dx.doi.org/10.1016/S1575-0973(06)75104-2

Pedrero-Pérez, E. J., López-Durán, A. y Olivar-Arroyo, A. (2011). Addiction: Frontal Personality Change but not Personality Disorder Comorbidity. Implications for Treatment of Addictive Disorders. En M. E. Jordan (Ed.), Personality Traits: Theory, Testing and Influences (pp. 1-36). New York: Nova Science Publishers.

Pedrero Pérez, E. J., Olivar Arroyo, Á. y Chicharro Romero, J. (2008). Cuestionario CAD-4: una medida biopsicosocial de la calidad de vida autopercibida en pacientes drogodependientes [CAD-4 Questionnaire: A Biopsychosocial Measure of Self-Perceived Quality of Life in Drug Addicts in Treatment]. Trastornos Adictivos, 10(1), 17-31. http://dx.doi.org/10.1016/S1575-0973(08)74543-4
Pedrero Pérez, E. J., Olivar Arroyo, Á. y Puerta García, C. (2007). El carácter como predictor de trastornos de la personalidad en adictos: la tipología caracterial de Cloninger y su abordaje psicosocial. Revista Española de Drogodependencias, 32(3), 342-369.

Pedrero Pérez, E. J., Pérez López, M., De Ena de la Cuesta, S y Garrido Caballero, C. (2005). Validación del Cuestionario de Variables de Interacción Psicosocial (VIP): hacia un modelo de tratamiento de las conductas adictivas "guiado por la personalidad" [Validation of the Psychosocial Interaction Variables Questionnaire: Towards a «Personality Guided Therapy» Model of Addiction Treatment]. Trastornos Adictivos, 7(3), 166-186. http://dx.doi.org/10.1016/S1575-0973(05)74523-2

Pedrero Pérez, E. J., Puerta García, C., Segura López, I. S. y Osorio del Río, S. O. (2004). Evolución de la sintomatología psicopatológica de los drogodependientes a lo largo del tratamiento [Evolution of Psicopathologic Symptoms of Drug Dependents Throughout the Treatment]. Trastornos Adictivos, 6(3), 176-191.

Pedrero Pérez, E. J., Rojo Mota, G. y Puerta García, C. (2008). Estilos de afrontamiento del estrés y adicción. Revista Española de Drogodependencias, 33(4), 256-270.

Pedrero Pérez, E. J., Rojo Mota, G. y Puerta García, C. (2009). Valores sociales y adicción a la cocaína: diferencias entre adictos en tratamiento y población no clínica [Validation of the Psychosocial Interaction Variables Questionnaire: Towards a «Personality Guided Therapy» Model of Addiction Treatment]. Revista Española de Drogodependencias, 34(2), 167-185. http://dx.doi.org/10.1016/S1575-0973(05)74523-2

Pedrero-Pérez, E. J., Ruiz-Sánchez de León, J. LozoyaDelgado, M. P., Rojo-Mota1, G., Llanero-Luque, M. y Puerta-García, C. (2015). La "Escala de Estrés Percibido": Estudio psicométrico sin restricciones en población no clínica y adictos a sustancias en 
tratamiento. Behavioral Psychology / Psicología Conductual, 23(2), 305-324.

Pfefferbaum, A., Adalsteinsson, E. y Sullivan, E. V. (2006). Supratentorial Profile of white Matter Microstructural Integrity in Recovering Alcoholic Men and Women. Biological Psychiatry, 59(4), 364-372.

http://dx.doi.org/10.1016/j.biopsych.2005.06.025

Rasmussen, H. N., Scheier, M. F. y Greenhouse, J. B. (2009). Optimism and Physical Health: A MetaAnalytic Review. Annals of Behavioral Medicine, 37(3), 239-256. http://dx.doi.org/10.1007/s12160009-9111-x

Remor, E. A. y Carrobles, J. A. (2001). Versión española de la Escala de estrés percibido (PSS-14): estudio psicométrico en una muestra VIH+. Ansiedad y Estrés, 7, 195-201.

Sánchez-López, M. P. y Dresch, V. (2008). The 12-Item General Health Questionnaire (GHQ-12): Reliability, External Validity and Factor Structure in the Spanish Population. Psicothema, 20(4), 839843.

Sariyska, R., Reuter, M., Bey, K., Sha, P., Li, M., Chen, Y. F., ... y Montag, C. (2014). Self-esteem, Personality and Internet Addiction: A CrossCultural Comparison Study. Personality and Individual Differences, 61, 28-33. http://dx.doi.org/10.1016/j.paid.2014.01.001

Serrano-Gallardo, P., Lizán-Tudela, L., Díaz-Olalla, J. M. y Otero, A. (2009). Valores poblacionales de referencia de la versión española de las viñetas COOP/WONCA de calidad de vida en población adulta urbana [Reference Population Values of the Spanish Version of the COOP/WONCA Charts of Quality of Life in an Urban Adult Population]. Medicina Clínica, 132, 336-343. http://dx.doi.org/10.1016/j.medcli.2008.04.009

Skevington, S. M., Sartorius, N., Amir, M. y THE WHOQOL Group. (2004). Developing Methods for
Assessing Quality of Life in Different Cultural Settings. Social Psychiatry and Psychiatric Epidemiology, 39 , $1-8$. 
